Article

\title{
Fabrication of Activated Carbon Fibers with Sheath-Core, Hollow, or Porous Structures via Conjugated Melt Spinning of Polyethylene Precursor
}

\author{
Jong Sung Won ${ }^{1}$, Ha Ram Lee ${ }^{2}$, Min Jun Lee ${ }^{2}$, Min Hong Jeon ${ }^{2}$, Seung Goo Lee ${ }^{2}$ and \\ Yong Lak Joo ${ }^{1, *(\mathbb{D})}$ \\ 1 Robert Frederick Smith School of Chemical and Biomolecular Engineering, Cornell University, \\ Ithaca, NY 14853, USA; jw2636@cornell.edu \\ 2 Department of Advanced Organic Materials \& Textile Engineering, Chungnam National University, \\ Daejeon 34134, Korea; haram523@cnu.ac.kr (H.R.L.); irai94@o.cnu.ac.kr (M.J.L.); \\ hong831@o.cnu.ac.kr (M.H.J.); lsgoo@cnu.ac.kr (S.G.L.) \\ * Correspondence: ylj2@cornell.edu
}

Received: 18 November 2020; Accepted: 30 November 2020; Published: 3 December 2020

\begin{abstract}
Using polyethylene as carbon precursor, we have fabricated cost-effective carbon fibers with a sheath-core structure via conjugate melt spinning. Low-density polyethylene (LDPE) and high-density polyethylene (HDPE) were used as the sheath and core of the fiber, respectively, while sulfonation with sulfuric acid was conducted to enable the crosslinking of polyethylene. We demonstrated that carbonization and activation of the sheath-core-structured polyethylene fiber can result in a well-developed microporous structure in the sheath layer, and due to the core-sheath structure, the resulting activated carbon fibers exhibit a high tensile strength of $\sim 455 \mathrm{MPa}$, initial modulus of $\sim 14.4 \mathrm{GPa}$, and Brunauer-Emmett-Teller (BET) surface area of $\sim 1224 \mathrm{~m}^{2} / \mathrm{g}$. Finally, activated carbon fibers with a hollow, sheath-core, and porous were successfully fabricated by controlling the degree of crosslinking of the LDPE/HDPE sheath-core fiber.
\end{abstract}

Keywords: activated carbon fiber; polyethylene precursor; hollow carbon fiber; sheath-core activated carbon fiber

\section{Introduction}

Activated carbon fiber is an important nano-porous carbon material used in filter applications because of its excellent adsorption properties. It has an intrinsic porous structure with low meso porosity and no macro porosity. Therefore, it has also been employed for heavy metal removal, as an adsorbent for hazardous volatiles, and for vapor sensing and gas storage [1-5]. The production of activated carbon fiber is not very different from that for carbon fiber. To date, many kinds of activated carbon fibers have been developed using carbon precursor fibers, such as polyacrylonitrile (PAN), pitch, rayon, and phenolic. These activated carbon fibers exhibit proper nano-porous structures and have been used for filtering hazardous volatile gases and very fine dusts-pollutants which have recently attracted global attention due to air pollution [6-11].

However, the typical cost of activated carbon fiber is much higher than that of other microporous materials, because of the cost of the precursor material and manufacturing process. This limit widespread usage of carbon fiber and activated carbon fibers. Therefore, recently, many efforts have focused on developing low-cost carbon fiber using low-cost precursor materials. One promising precursor material is polyethylene, which has the advantages of a very low cost and easy processing via conventional melt spinning [12-17]. 
Recently, some studies have reported successful manufacturing of carbon fiber from a polyethylene precursor. To prepare carbon fiber using a polyethylene precursor, sulfonation with sulfuric acid is typically performed to stabilize the precursor. This results in slightly poorer mechanical properties compared to conventional carbon fibers. Nevertheless, it provides novel routes for carbon fiber processing with a low-cost precursor. However, there have been almost no studies on developing activated carbon fiber using a polyethylene precursor. A polyethylene-based activated carbon fiber would be an important absorbent material with low cost and versatility [18-25].

On the other hand, the poor mechanical properties of most activated carbon fibers with a microporous structure make it very difficult to handle or treat for various applications. Lack of mechanical strength limits their usage in an unloaded state. Therefore, the fibers cannot be easily applied for a fabric structure which requires extensional properties and mechanical endurance [26,27].

In the current study, we fabricated activated carbon fiber using polyethylene fiber via conjugate spinning. Thus, a sheath-core structure of the precursor fiber was obtained, yielding better mechanical properties than those of conventional monoaxial activated carbon fibers. Two types of polyethylene, high-density (HDPE) and low-density (LDPE), were used as the core and sheath materials, respectively. HDPE in the core can maintain the mechanical strength of the fiber, while LDPE, with its low compactness in the sheath, can be activated easily. This unique bilateral fiber structure makes it possible to obtain a fully activated sheath layer and less-activated core layer with proper mechanical strength. In this study, the effects of the draw ratio of the precursor fiber on the sulfonation based stabilization process were investigated. After carbonization and activation of the polyethylene fiber, the nano-porous structure with a high pore area in resulting carbon fibers was analyzed. Finally, the mechanical properties of the prepared activated carbon fiber were tested to evaluate the effectiveness of the sheath-core fiber structure.

\section{Materials and Methods}

\subsection{Materials}

LDPE (XJ700, Lotte Chemical Co., Daejeon, Korea) was chosen as the sheath component and HDPE (2600F, Lotte Chemical Co., Daejeon, Korea) was used for the core of the sheath-core fibers.

\subsection{Preparation of Sheath-Core Fibers}

LDPE/HDPE sheath-core fibers were prepared by conjugate melt-spinning at $205^{\circ} \mathrm{C}$ at a take-up speed of $850 \mathrm{~m} / \mathrm{min}$, as shown schematically in Figure 1a. The content ratio of LDPE/HDPE in the sheath-core fiber was set to 50:50 by weight percent, as seen the scanning electron microscopy (SEM) image, Figure $1 b$. The drawing treatment of LDPE/HDPE sheath-core fibers was performed using a self-made drawing machine, at draw ratios of $1.2,1.3,1.5$, and 1.6 at $95{ }^{\circ} \mathrm{C}$.

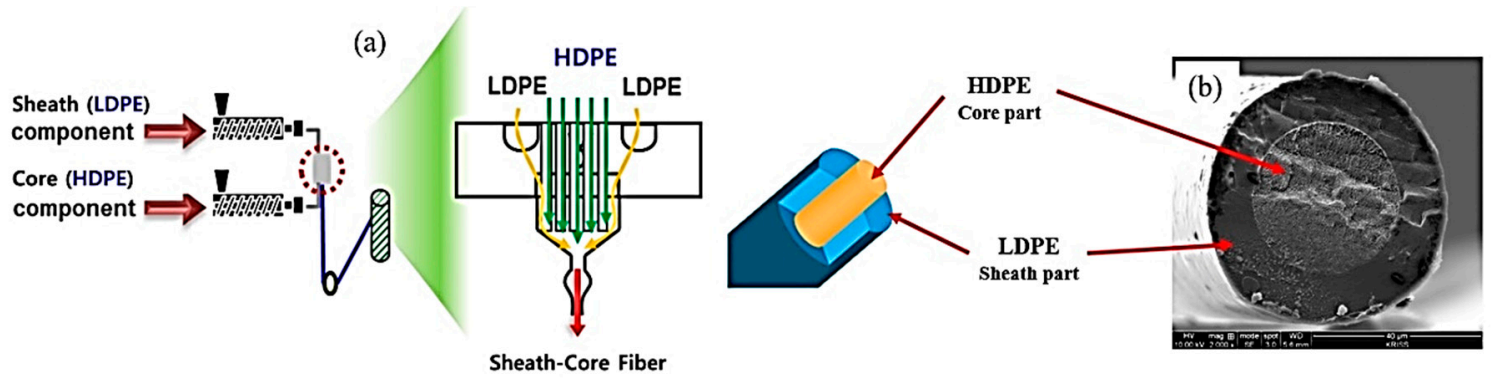

Figure 1. (a) Schematic melt spinning of sheath-core fiber and (b) SEM image of LDPE/HDPE sheath-core fiber manufactured by a conjugate melt-spinning. 


\subsection{Cross-Linking, Carbonization, and Activation of Sheath-Core Fibers}

The obtained precursors were stabilized through a dipping treatment in sulfuric acid $\left(\mathrm{H}_{2} \mathrm{SO}_{4}, 95 \%\right.$, Samchun Co., Seoul, Korea) as shown in Figure 2a. Figure $2 b$ shows the shape of the sulfated fibers under loads of $0,0.25$ and $0.5 \mathrm{MPa}$. When a load of $0.5 \mathrm{MPa}$ was applied, it was confirmed that the fiber was cut. Therefore, in this study, sulfuric acid treatment was carried out with a load of $0.25 \mathrm{MPa}$. Uniaxially aligned LDPE/HDPE sheath-core fibers were stabilized by dipping, with a load of $0.25 \mathrm{MPa}$ applied at different temperatures and times, as shown in Table 1. Carbonization was performed in nitrogen atmosphere at a final temperature of $900{ }^{\circ} \mathrm{C}$ and a heating rate of $5{ }^{\circ} \mathrm{C} / \mathrm{min}$. The process time at the maximum temperature was set as $5 \mathrm{~min}$ to complete carbonization. After completion of carbonization, the furnace was turned off and maintained in nitrogen atmosphere. This condition of inert atmosphere was maintained until the temperature inside the furnace reactor reached room temperature. Activated sheath-core carbon fibers were prepared by chemical activation. Activation of the cross-linked LDPE/HDPE sheath-core fibers was carried out by dipping for $24 \mathrm{~h}$ using a 1-4 M KOH solution and drying for $12 \mathrm{~h}$ in a convection oven at $100{ }^{\circ} \mathrm{C}$.
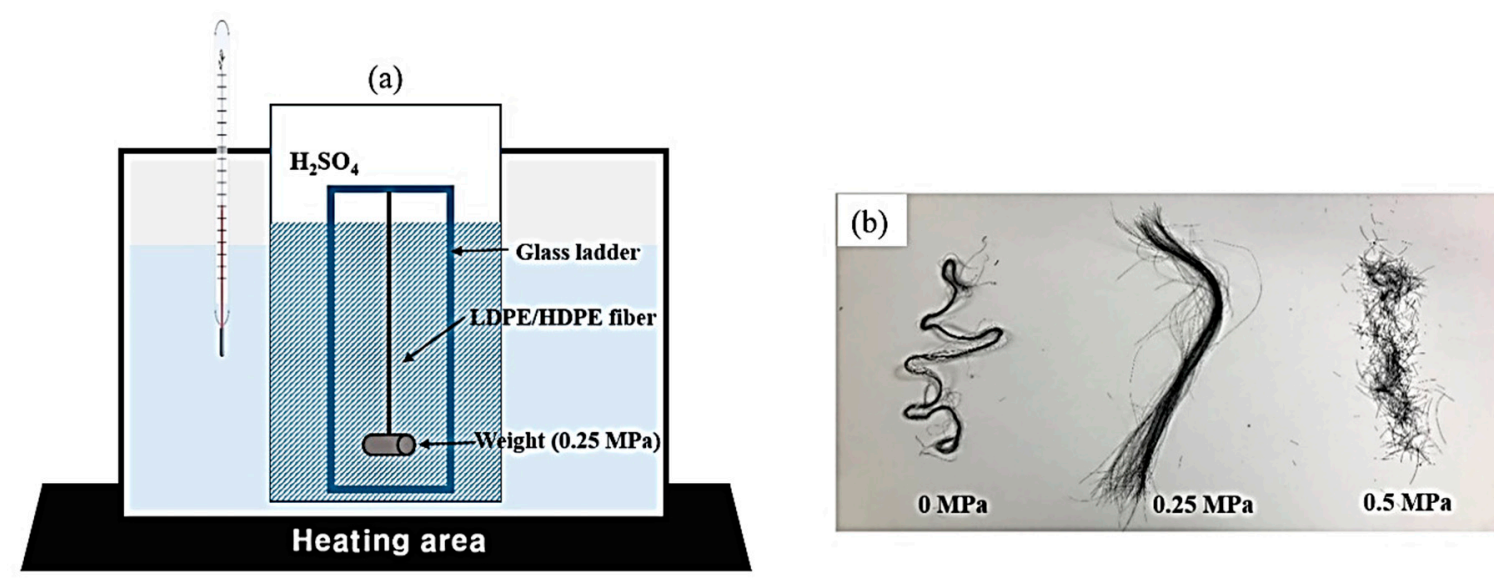

Figure 2. (a) Concept illustration of the crosslinking process and (b) images of the sulfated fibers under loads of $0,0.25$ and $0.5 \mathrm{MPa}$ for the precursor fibers.

Table 1. Sulfuric acid treatment conditions of LDPE/HDPE sheath-core fiber.

\begin{tabular}{cc}
\hline Temperature $\left({ }^{\circ} \mathbf{C}\right)$ & $\mathbf{1 2 0 , 1 3 0 , 1 4 0 , 1 5 0}$ \\
\hline Time $(\mathrm{Min})$ & $60,90,120,150$ \\
Loading $(\mathrm{MPa})$ & 0.25 \\
\hline
\end{tabular}

\subsection{Characterization of Sheath-Core Fibers}

The thermal transition behavior of the LDPE/HDPE sheath-core fibers was measured by differential scanning calorimetry (DSC, Mettler-Toledo DSC1) in the temperature range of $25-250^{\circ} \mathrm{C}$ at a heating rate of $10^{\circ} \mathrm{C} / \mathrm{min}$ in nitrogen atmosphere. The melting enthalpy was measured and the aromatization index (AI) was calculated to identify the level of cross-linking, using the following Equation (1) [18,28]:

$$
\text { Aromatization index }(\%)=\left(\frac{\Delta H_{0}}{\Delta H / \Delta H_{0}}\right) \times 100
$$

where $\Delta H_{0}$ is the melting enthalpy of the pristine sample and $\Delta H$ is the melting enthalpy of the cross-linked sample.

The effects of the temperature characteristics of the cross-linking processes were investigated using thermogravimetric analysis (TGA, Mettler-Toledo TGA/DSC1). In TGA, all cross-linked samples were heated from $25^{\circ} \mathrm{C}$ to $800^{\circ} \mathrm{C}$ at a heating rate of $10^{\circ} \mathrm{C} / \mathrm{min}$ in a nitrogen atmosphere. 
The surface and cross-sectional morphological features of the LDPE/HDPE sheath-core fibers after cross-linking and carbonization were characterized by SEM (S4700, HITACHI, Daejeon, Korea). To obtain cross-sectional SEM images, the samples were immersed in a liquid nitrogen bath for $5 \mathrm{~min}$ and then sectioned using a diamond cutter.

A Fourier transform infrared (FT-IR) spectrophotometer (Bruker Optic GmbH, ALPHA-P, Daejeon, Korea) equipped with an attenuated total reflectance (ATR) accessory was used to examine the surface composition changes in the fibers attributed to cross-linking treatment. The spectra were recorded in the transmission mode in the range of $3500-500 \mathrm{~cm}^{-1}$, with a spectral resolution of $4 \mathrm{~cm}^{-1}$, and accumulation of 128 scans for a high signal-to-noise ratio.

The tensile mechanical tests were carried out using a universal tensile testing machine (Model 4467, Instron) at a crosshead speed of $1 \mathrm{~mm} / \mathrm{min}$ for the cross-linked sheath-core fibers and carbon fibers (ASTM D3822).

The Brunauer-Emmett-Teller (BET) method was applied to determine the total surface area, while the pore size distribution and micropore volume were estimated by applying the Barret-Joyner-Halenda (BJH) method and the non-local density functional theory (NLDFT) over the adsorption isotherm.

\section{Results and Discussion}

\subsection{Characterization of LDPE/HDPE Sheath-Core Fibers}

Figure 3 shows the cross-sectional SEM images of the LDPE/HDPE sheath-core fibers prepared at different draw ratios. It is observed that the diameters of the sheath fibers as well as core fibers decrease with increasing draw ratio. We note that the interface between HDPE core and LDPE shear is smooth and compact, and the LDPE sheath is well packed in the sheath-core fibers manufactured at a draw ratio of 1.2-1.5, whereas the interface becomes less uniform in those manufactured at the higher draw ratio of 1.6, which may be attributed to the interfacial instability at high drawing speeds. Nonetheless, the ratio of core radius to shear layer thickness remains the same at about 0.5 for all draw ratios studied.
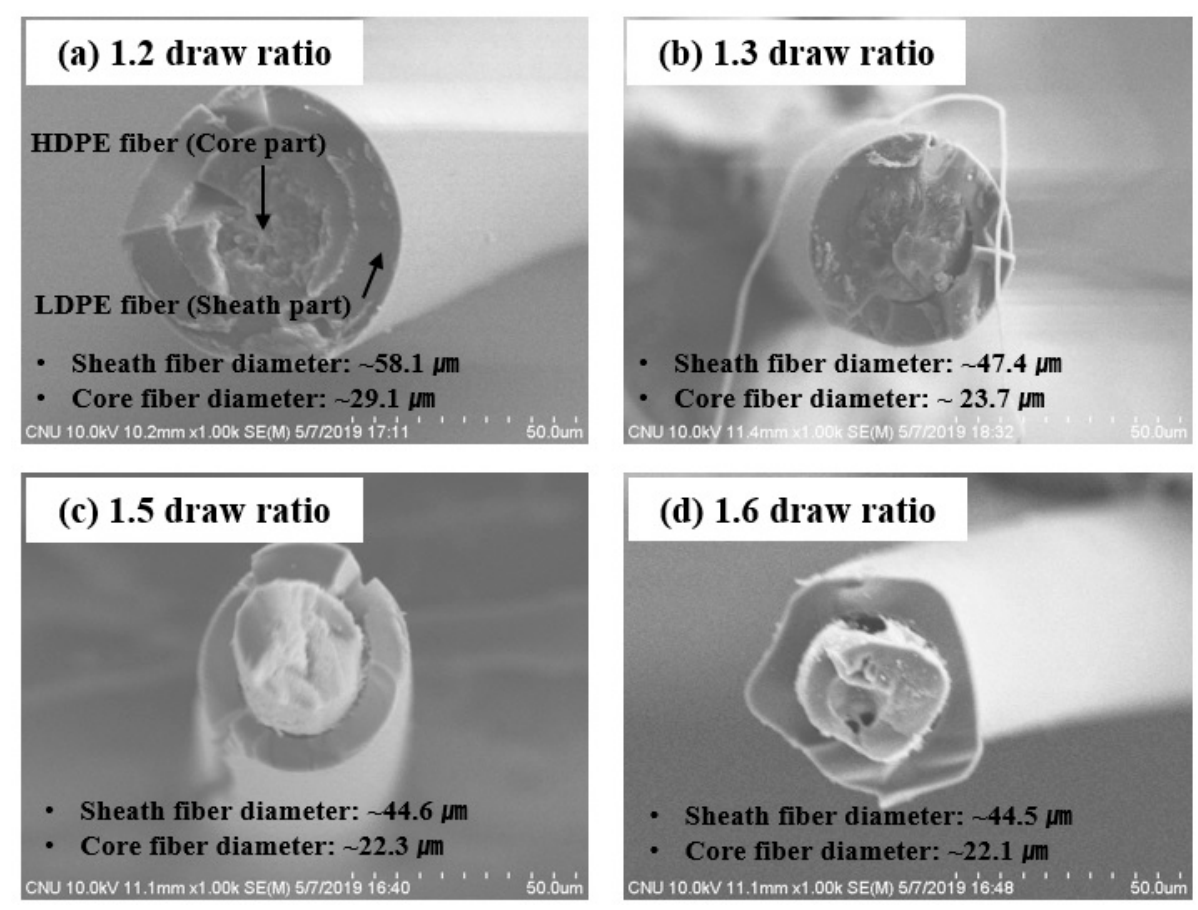

Figure 3. Cross-sectional SEM image of LDPE/HDPE sheath-core fibers manufactured at different draw ratio of 1.2-1.6 times. 
Next, the tensile mechanical properties of the LDPE/HDPE sheath-core fibers manufactured at different draw ratios were investigated. The tensile strength and initial modulus of the fibers increases with increasing the draw ratio up to 1.5, as shown in Figure 4. Beyond a ratio of 1.5, however, both tensile strength and modulus decrease, possibly due to the less uniform core and sheath morphology and less circular cross section of the fibers. Therefore, LDPE/HDPE sheath-core fibers manufactured at the draw ratio of 1.5 were used to obtain the activated carbon fibers.

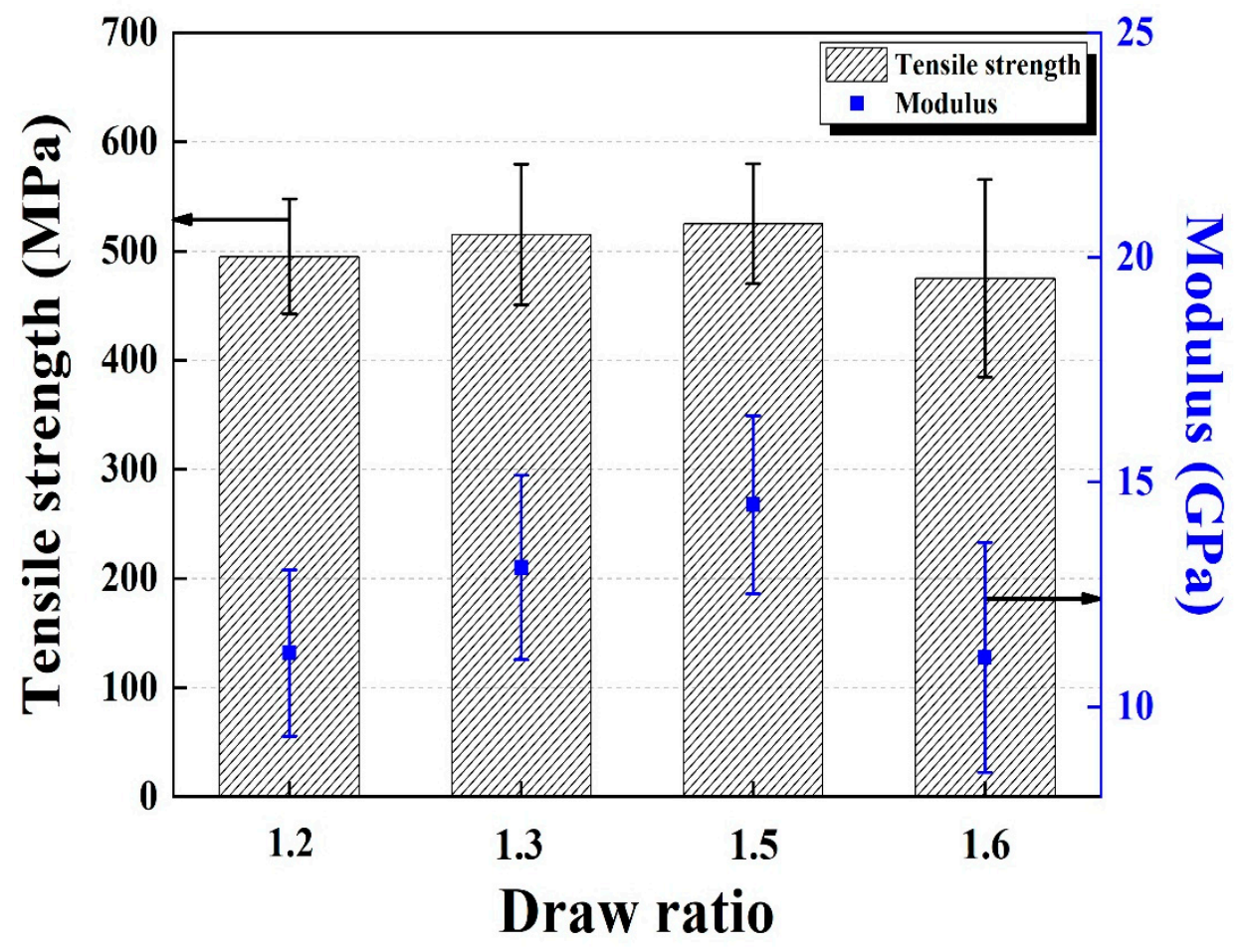

Figure 4. Tensile strength and initial modulus of LDPE/HDPE sheath-core fibers manufactured at different at draw ratio of 1.2-1.6 times.

\subsection{Characterization of Cross-Linked LDPE/HDPE Sheath-Core Fibers}

The LDPE/HDPE sheath-core fibers were cross-linked by sulfuric acid. Figure 5 shows the SEM images of the cross-section of the cross-linked LDPE/HDPE sheath-core fibers manufactured at different cross-linking temperatures $\left(120-150^{\circ} \mathrm{C}\right)$ and time (60-150 min). The range of cross-linking temperature was determined based on the melting behavior of two components: the melting transition temperature (Tm) of the LDPE sheath component in the sheath-core fibers was in the range of $96-110^{\circ} \mathrm{C}$, which was lower than that $\left(123-133^{\circ} \mathrm{C}\right)$ of the HDPE core component. For the cross-linked sheath-core fibers manufactured at cross-linking temperatures of 120 and $130{ }^{\circ} \mathrm{C}$ under a constant load of $0.25 \mathrm{MPa}$ for $120 \mathrm{~min}$ (Figure 5(a1,a2)), good interaction among the sheath-core fibers as well as good structure of the sheath-core component was achieved. However, the LDPE sheath part melts at $140-150{ }^{\circ} \mathrm{C}$, to which the core exhibit defects in the final sheath-core fiber (Figure 5(a3,a4)). For cross-linked sheath-core fibers manufactured at different cross-linking durations of 60-150 min under a constant cross-linking temperature of $130{ }^{\circ} \mathrm{C}$ and load of $0.25 \mathrm{MPa}$ (Figure 5(b1-b4)), good interaction and shape of the sheath-core fibers are attained below $120 \mathrm{~min}$, whereas the structure of the LDPE sheath part exhibits visible fractures above $150 \mathrm{~min}$ (Figure 5(b4)).

Figure 6 shows the DSC heating thermograms of the LDPE/HDPE sheath-core fibers cross-linked for 60-150 min under constant pressure and temperature of $0.25 \mathrm{MPa}$ and $130^{\circ} \mathrm{C}$, respectively. In the first DSC heating curves at the bottom, double-melting endotherms are observed for the untreated LDPE/HDPE sheath-core fiber. The lower endotherm at $\sim 102{ }^{\circ} \mathrm{C}$ is associated with the melting of the sheath-part LDPE, whereas the higher endotherm at $\sim 128^{\circ} \mathrm{C}$ is associated with the melting of 
the core-part HDPE. For the sheath-core fiber cross-linked for greater than $60 \mathrm{~min}$, only a single endothermic peak appears at $\sim 130{ }^{\circ} \mathrm{C}$, because the cross-linking of LDPE has completed. The lower melting enthalpy for the sheath-core fiber cross-linked for $150 \mathrm{~min}$ is attributed to the increased cross-linking of the HDPE core part in the LDPE/HDPE sheath-core fiber, compared to that cross-linked for $60 \mathrm{~min}$. This indicates that the LDPE/HDPE sheath-core fiber is rendered infusible by the sulfuric acid treatment.
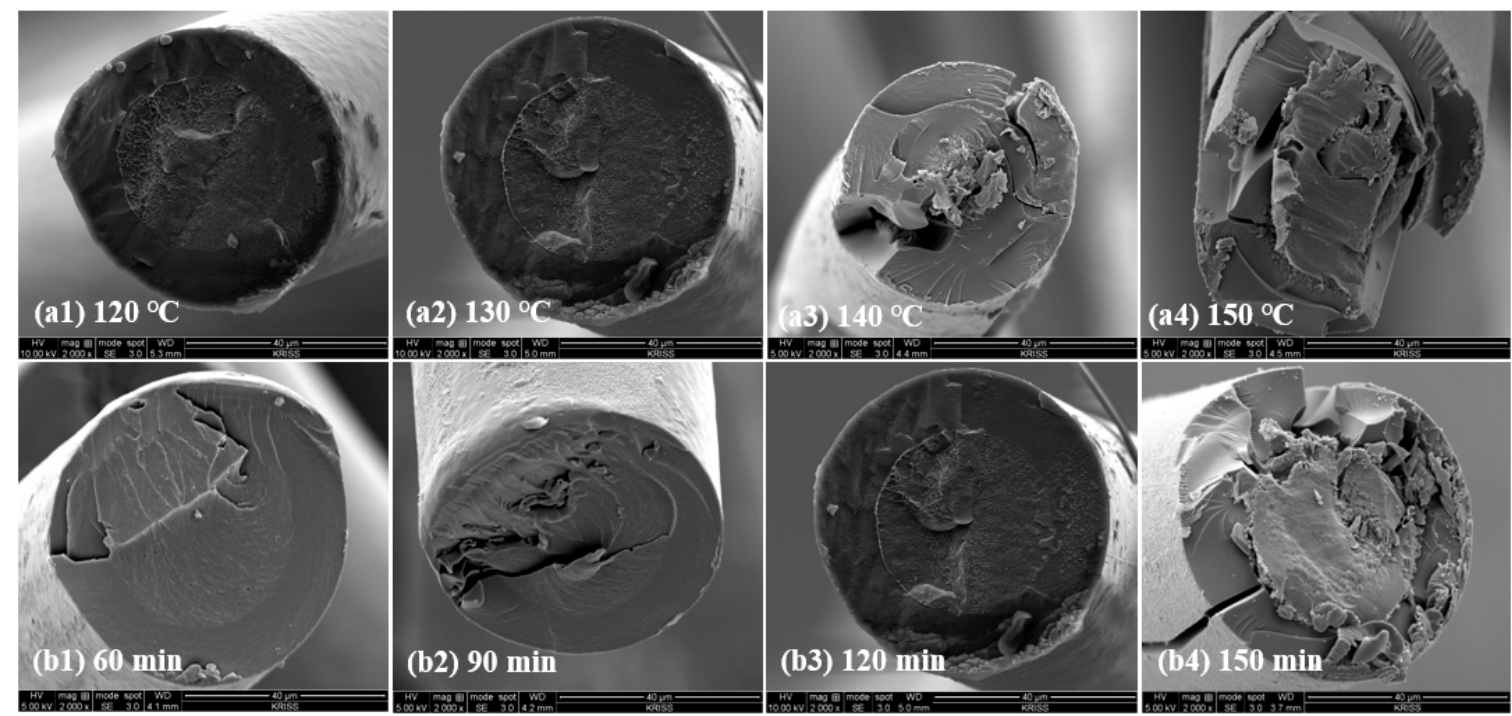

Figure 5. SEM images of the sulfonated LDPE/HDPE sheath-core fibers at various crosslinking temperature and time: (a1-a4) temperatures of $120-150{ }^{\circ} \mathrm{C}$ under $0.25 \mathrm{MPa}$ for $120 \mathrm{~min}$; (b1-b4) duration times of $60-150 \mathrm{~min}$ under $0.25 \mathrm{MPa}$ at $130^{\circ} \mathrm{C}$.

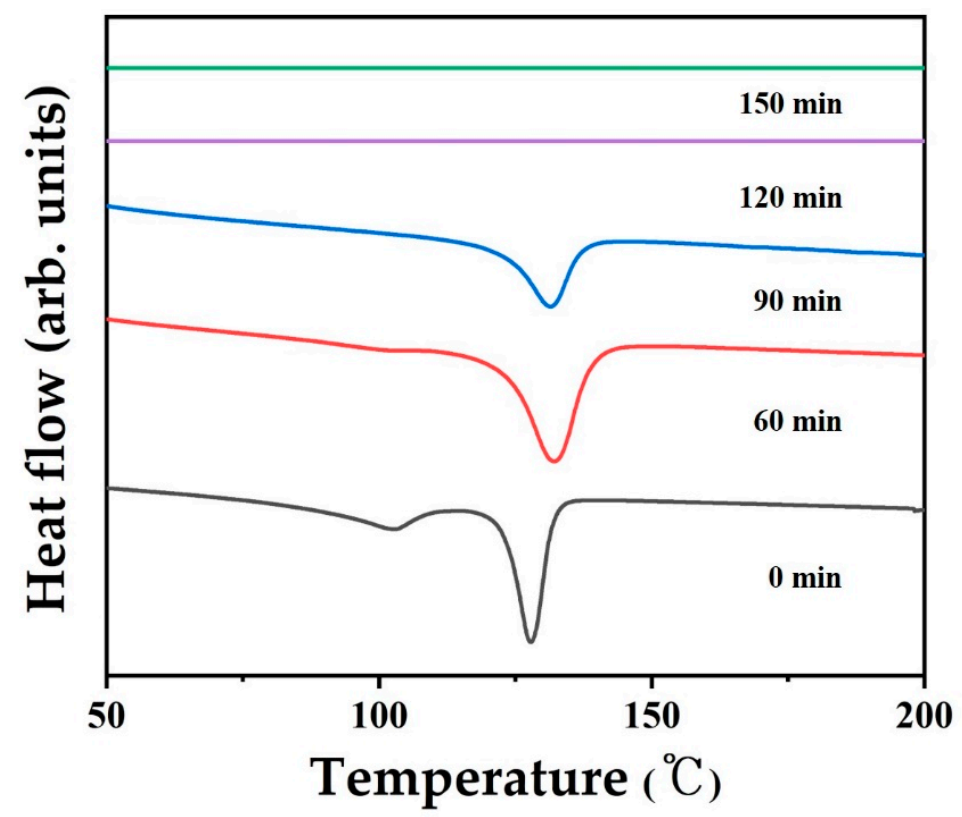

Figure 6. DSC patterns of the sulfonated fibers for various crosslinking time at $130{ }^{\circ} \mathrm{C}$.

Table 2 lists the aromatization index (AI) values calculated from the melting enthalpy experiments. During the acid treatment at $130^{\circ} \mathrm{C}$, the melting enthalpy of core-part HDPE decreased from $215.6 \mathrm{~J} / \mathrm{g}$ to $120.9,69.1$ and $0 \mathrm{~J} / \mathrm{g}$ at a cross-linking time of $60,90,120$, and $150 \mathrm{~min}$, respectively, corresponding to AI values of $43.9 \%, 67.95 \%$ and $100 \%$. The melting enthalpy of sheath-part LDPE decreased from 
$48.5 \mathrm{~J} / \mathrm{g}$ to 3.9 , and $0 \mathrm{~J} / \mathrm{g}$ at a cross-linking time of $60,90,120$, and $150 \mathrm{~min}$, respectively, corresponding to AI values of $91.88 \%$ and $100 \%$. The LDPE sheath part becomes completely infusible after $60 \mathrm{~min}$, while the HDPE core part becomes infusible at an AI of $100 \%$ at $120 \mathrm{~min}$. Therefore, from this result, it was confirmed that the crosslinking of the HDPE core part required a longer sulfonation time than the LDPE sheath part. As demonstrated in the later section, this difference in crosslinking behavior by sulfonation of LDPE and HDPE can be utilized in fabricating hollow porous carbon fibers.

Table 2. Aromatization index values of LDPE/HDPE sheath-core fibers for various crosslinking time at $130{ }^{\circ} \mathrm{C}$.

\begin{tabular}{|c|c|c|c|c|c|c|}
\hline \multicolumn{2}{|c|}{ Crosslinking Time (min) } & \multirow{2}{*}{$\begin{array}{l}\mathbf{0} \\
0\end{array}$} & \multirow{2}{*}{$\begin{array}{c}\mathbf{6 0} \\
43.9\end{array}$} & \multirow{2}{*}{$\begin{array}{c}90 \\
67.95\end{array}$} & \multirow{3}{*}{$\begin{array}{l}\mathbf{1 2 0} \\
100\end{array}$} & \multirow{2}{*}{$\begin{array}{l}\mathbf{1 5 0} \\
100\end{array}$} \\
\hline Crosslink & HDPE & & & & & \\
\hline percentage $(\%)$ & LDPE & 0 & 91.88 & 100 & & 100 \\
\hline
\end{tabular}

Next, FTIR was used to characterize the changes of chemical structure of the LDPE/HDPE sheath-core fibers during cross-linking. Figure 7 shows the FTIR spectra of the LDPE/HDPE sheath-core fibers cross-linked for $60-150 \mathrm{~min}$ at $130^{\circ} \mathrm{C}$. Bands at $3000-2850 \mathrm{~cm}^{-1}, 1384-1475 \mathrm{~cm}^{-1}$, and $710-750 \mathrm{~cm}^{-1}$ appear in all samples, and can be attributed to the stretching of the $\mathrm{C}-\mathrm{H}$ bond in methylene groups and the $\mathrm{C}-\mathrm{H}$ long-chain bond in LDPE and HDPE, respectively. With increasing duration of cross-linking, the peak for the $-\mathrm{CH}_{2}$ bond at $1465 \mathrm{~cm}^{-1}$ shifts to $1280-1080 \mathrm{~cm}^{-1}$ and $1040 \mathrm{~cm}^{-1}$, respectively. The peak intensities near $724 \mathrm{~cm}^{-1}$ (C-H stretching vibrations) was prominent in the pure LDPE/HDPE sheath-core structure. In succession, these were found to reduction with increasing sulfonation time. The peak intensities diminished evidently with enhanced duration and sulfonate group incorporation in the sulfonated LDPE/HDPE sheath-core fibers. This shows that sulfonation was achieved by $\mathrm{O}=\mathrm{S}=\mathrm{O}$ stretching vibrations. Therefore, the H-branch on the LDPE and HDPE chain was substituted for the sulfonic acid group of $-\mathrm{HSO}_{3}$ and bound to another $\mathrm{H}$-branch to form the sulfone of $\mathrm{SO}_{2}$. In addition, with increasing duration of cross-linking, the composition of carbon and hydrogen decreased while the composition of oxygen and sulfur increased. The decrease in composition of carbon and hydrogen indicates a decrease in the number of $\mathrm{C}-\mathrm{H}$ bonds, whereas the increase in the absorption for a sulfone group $\left(\mathrm{SO}_{2}\right)$ refers to an increase in the composition of oxygen and sulfur (see Supplementary Table $\mathrm{S} 1$ ).

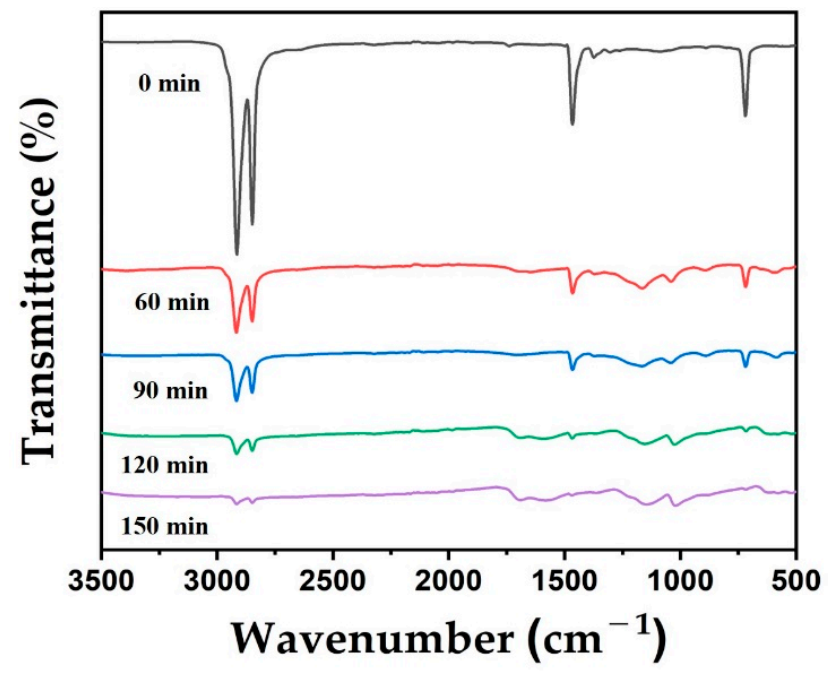

Figure 7. FTIR patterns of the sulfonated LDPE/HDPE sheath-core fibers for various crosslinking time at $130{ }^{\circ} \mathrm{C}$.

Figure 8 shows the increasing weight retention with increasing cross-linking duration. TGA curves indicate that the decomposition temperatures for all LDPE/HDPE sheath-core fibers cross-linked via sulfuric acid lie in the range of $490-550^{\circ} \mathrm{C}$. The thermal stability, based on the polymer decomposition temperature and integral procedural decomposition temperature, as well as the carbonization yield 
increases with increasing cross-linking duration. The cross-linking reaction improves the decomposition stability and heat exhaustion, resulting from the most densely cross-linked structure at $150 \mathrm{~min}$. This also enhances gel structures in the LDPE/HDPE sheath-core fibers, resulting in high carbonization yields. The LDPE/HDPE sheath-core fibers cross-linked at $150 \mathrm{~min}$ exhibit the highest weight retention $(47.72 \%)$ among all the fibers. In addition, the flammability of the stabilized samples was determined by a separate burning test that samples were put on flame from a blow torch. The samples that do not burn in general can be carbonized without burning at higher temperature. As shown in burning test images in Supplementary Figure S1, the LDPE/HDPE sheath-core fibers cross-linked at 120-150 min did not burn, whereas the LDPE/HDPE sheath-core fibers cross-linked at 0-90 min burned in the flame. This also confirms that after 120 min of sulfonation, the LDPE/HDPE sheath-core fibers were effectively cross-linked.

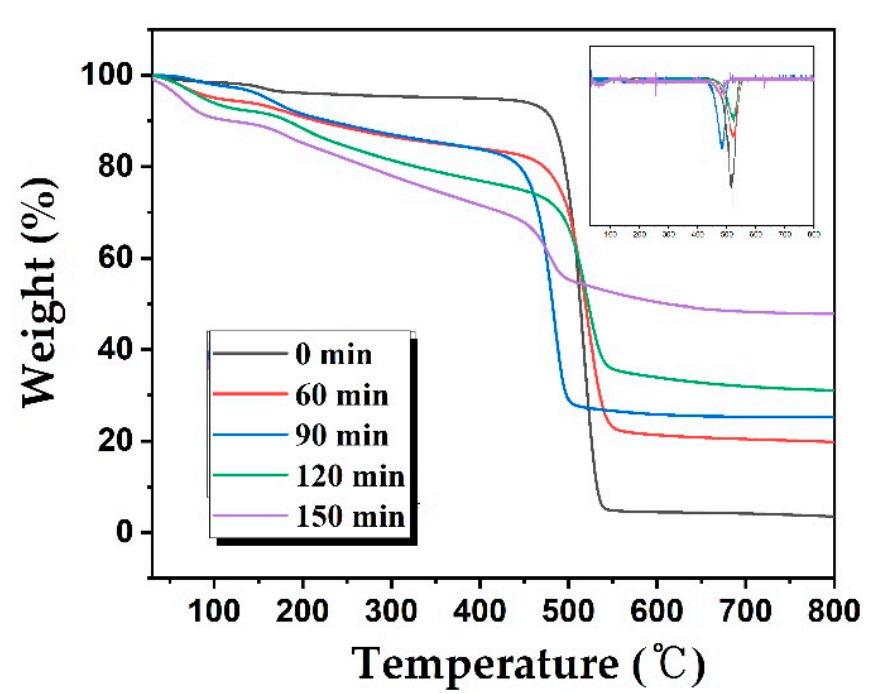

Figure 8. TGA results of the sulfonated LDPE/HDPE sheath-core fibers for various crosslinking times at $130{ }^{\circ} \mathrm{C}$. The inset is corresponding differential curves for various crosslinking times at $130{ }^{\circ} \mathrm{C}$.

Figure 9 shows the change in density of LDPE/HDPE sheath-core fibers for various cross-linking times at $130{ }^{\circ} \mathrm{C}$. It is observed that an increase in cross-linking time resulted in an increase in density from 0.9365 to $1.5191 \mathrm{~g} / \mathrm{cm}^{3}$ for LDPE/HDPE sheath-core fibers until cross-linking duration of $120 \mathrm{~min}$.

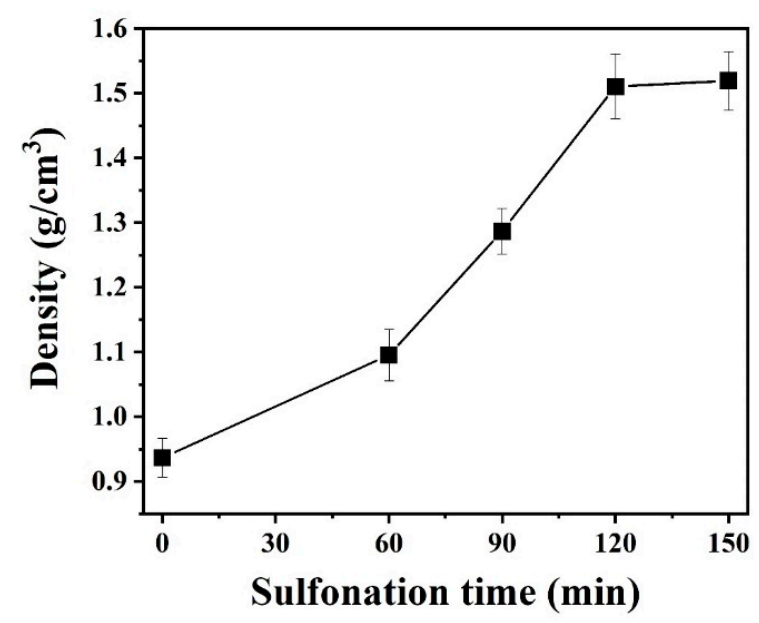

Figure 9. Density of the sulfonated LDPE/HDPE sheath-core fibers for various crosslinking times at $130{ }^{\circ} \mathrm{C}$. 


\subsection{Characterization of Carbonized and Activated LDPE/HDPE Sheath-Core Fibers}

The external pore structures of the carbonized and activated LDPE/HDPE sheath-core carbon fibers are presented via SEM images in Figure 10. It is observed that the morphological features of $\mathrm{KOH}$-activated LDPE/HDPE sheath-core carbon fibers are strongly influenced by $\mathrm{KOH}$ concentration (Figure 10a-d). The structure and distribution of pores are very non-uniform possibly due to the fast reaction of KOH. SEM images of the carbonized LDPE/HDPE sheath-core fibers at 1 molarity also indicate the presence of relatively smooth surface and few pores (Figure 10a). On the other hand, the activated LDPE/HDPE sheath-core carbon fibers at 2-4 molarity exhibit surfaces containing macro, meso, micro pores, with increased macro pores at high $\mathrm{KOH}$ concentration. As the $\mathrm{KOH}$ concentration increases, highly cracked and collapsed surfaces are obtained, indicating the violent reaction between $\mathrm{KOH}$ and surface carbon with increasing $\mathrm{KOH}$ amount. This demonstrates that proper $\mathrm{KOH}$ treatment can lead to the creation of micro pores and high surface areas.
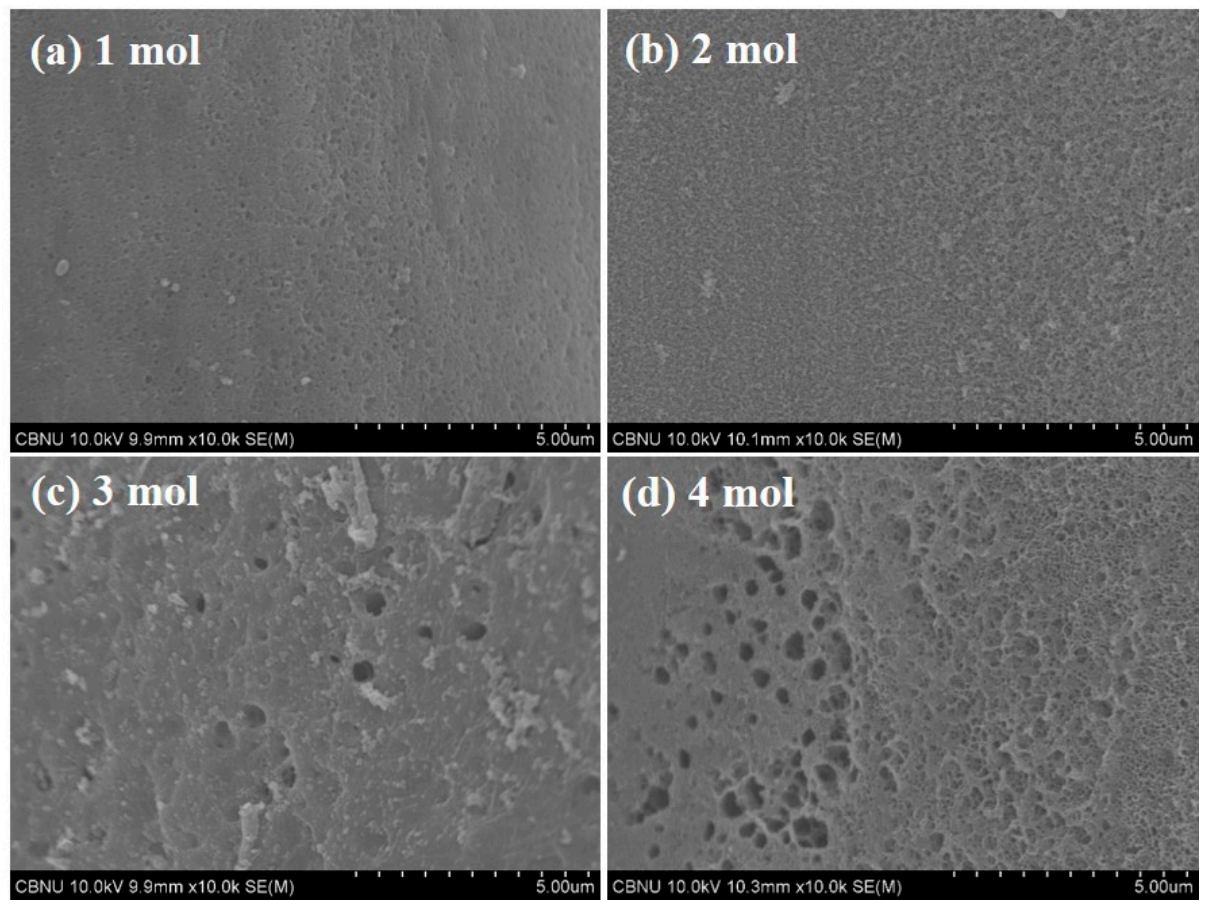

Figure 10. SEM images of the carbonized and activated LDPE/HDPE sheath-core fibers at various $\mathrm{KOH}$ molarity: (a) $1 \mathrm{~mol}$; (b) $2 \mathrm{~mol}$; (c) $3 \mathrm{~mol}$; (d) 4 mol.

To quantify the effect of $\mathrm{KOH}$ on the pore structure and surface area, we present the $\mathrm{BJH}$ incremental pore area distributions of the activated LDPE/HDPE sheath-core carbon fibers at four different $\mathrm{KOH}$ concentration in Figure 11. The nitrogen isotherm for the activated LDPE/HDPE sheath-core carbon fibers provided important information on the specific surface area and pore. It is observed that $\mathrm{KOH}$ chemical activation of the carbon fiber increases the surface area and porosity. The activated LDPE/HDPE sheath-core carbon fibers at 3-4 molarity exhibit better properties, with a BET surface area of $1224.36 \mathrm{~m}^{2} / \mathrm{g}$ and $1170.66 \mathrm{~m}^{2} / \mathrm{g}$, respectively, compared with those at 1-2 molarity $\left(661.55 \mathrm{~m}^{2} / \mathrm{g}\right.$ and $889.38 \mathrm{~m}^{2} / \mathrm{g}$, respectively). Thus, these results indicate that these carbon fibers activated by $\mathrm{KOH}$ are predominantly populated with micro pores. However, it was confirmed that when the molar ratio of $\mathrm{KOH}$ activation is very high, not only micro pores but also meso and macro pores are formed. Surface area analysis by BET show that the carbon fibers activated with 1 molarity to 4 molarity $\mathrm{KOH}$ had various surface properties in terms of the surface area and pores.

Results for the tensile mechanical tests of the LDPE/HDPE sheath-core carbon fibers after activation are presented in Figure 12. Generally, tensile strength increases by carbonization and decreases after activation. The decrease in tensile strength with activation exhibits different strengths depending on 
the pore size and shape formed on the fiber surface. However, the strength of $455 \mathrm{MPa}$ obtained by conjugate spinning of the sheath-core structure in the current study is regarded to be significantly higher than a single polyethylene based activated carbon fiber [29]. This improvement in tensile strength after carbonization and activation can be attributed to the bicomponent structure of the fiber, with a HDPE core and LDPE sheath, compared to conventional single-structured carbon fiber. This unique bilateral fiber structure enables the full activation of the sheath layer with lesser activation of the core layer, to provide suitable mechanical strength.
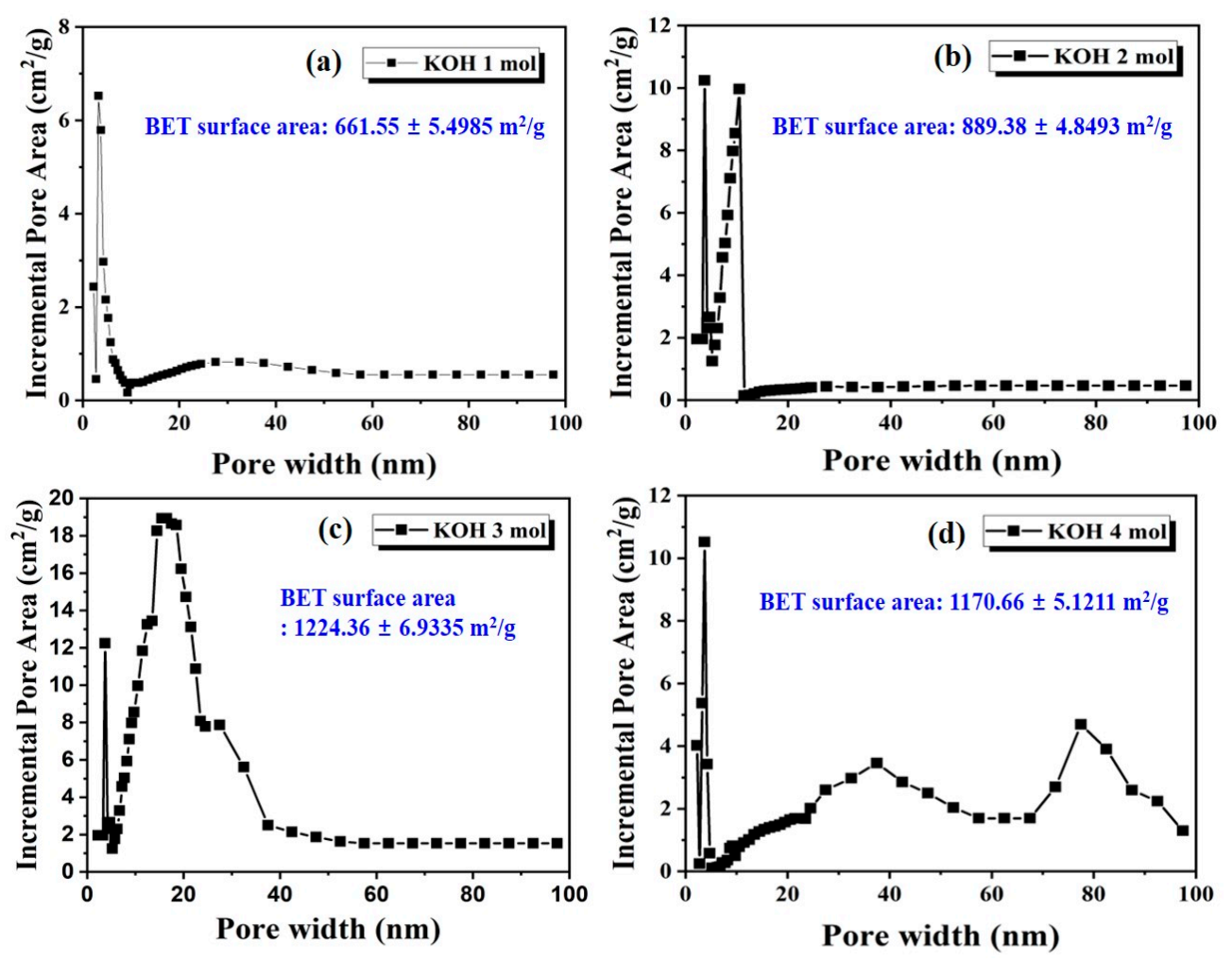

Figure 11. BJH analysis of nitrogen physisorption showing the incremental pore area distribution of activated LDPE/HDPE sheath-core carbon fibers at various $\mathrm{KOH}$ molarity: (a) 1 mol; (b) 2 mol; (c) $3 \mathrm{~mol}$; (d) 4 mol.

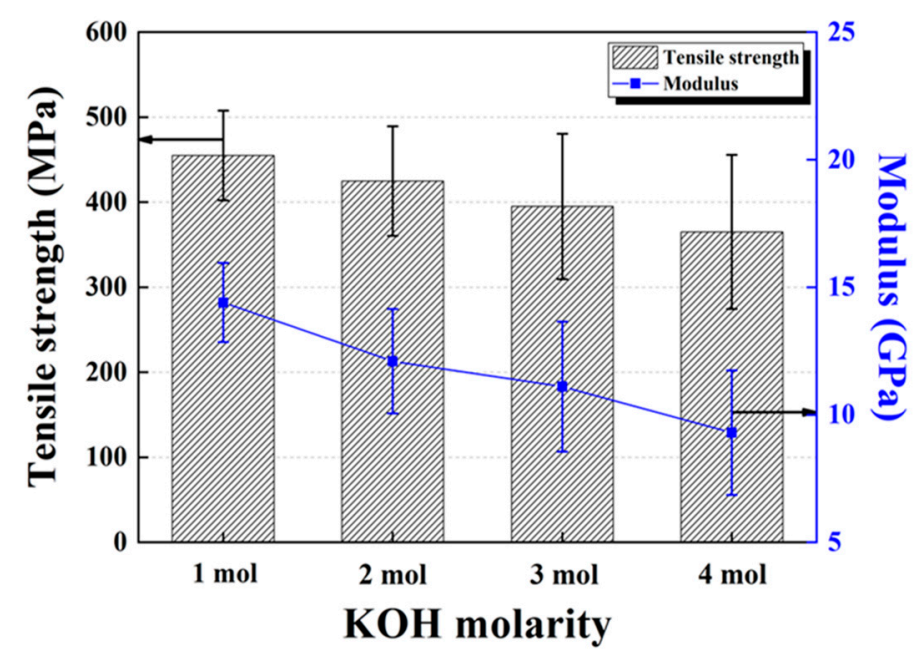

Figure 12. Tensile strength and initial modulus of activated LDPE/HDPE sheath-core carbon fibers at various $\mathrm{KOH}$ molarity. 
Finally, Figure 13 shows cross section SEM images after carbonization and activation of the sulfonated LDPE/HDPE sheath-core fibers for time of 60-120 min under $0.25 \mathrm{MPa}$ at $130^{\circ} \mathrm{C}$. As a result, cross sections of activated carbon fibers having three different structures were confirmed. It should be noted that for the LDPE/HDPE sheath-core carbon fibers sulfonated at cross-linking time of $60 \mathrm{~min}$ (Figure 13a), a cross section of the activated carbon fiber having a large hollow structure was observed. The difference in cross-linking in HDPE core and LDPE sheath and thus incomplete cross-linking of the HDPE core and inner region of the LDPE sheath went through thermal decomposition during the carbonization process to form a large pore structure. On the other hand, as the sulfonation time increased to $90 \mathrm{~min}$, the hollow structure became smaller due to the enhanced degree of cross-linking of the entire LDPE sheath while cross-linking of the core HDPE is still incomplete. In the case of activated sheath-core carbon fibers with $100 \%$ crosslinking of both LDPE sheath and HDPE core for 120 min sulfonation, a hollow structure was not observed as shown in Figure 13c, and the coherent core structure that could contribute to the improvement of tensile properties was observed.

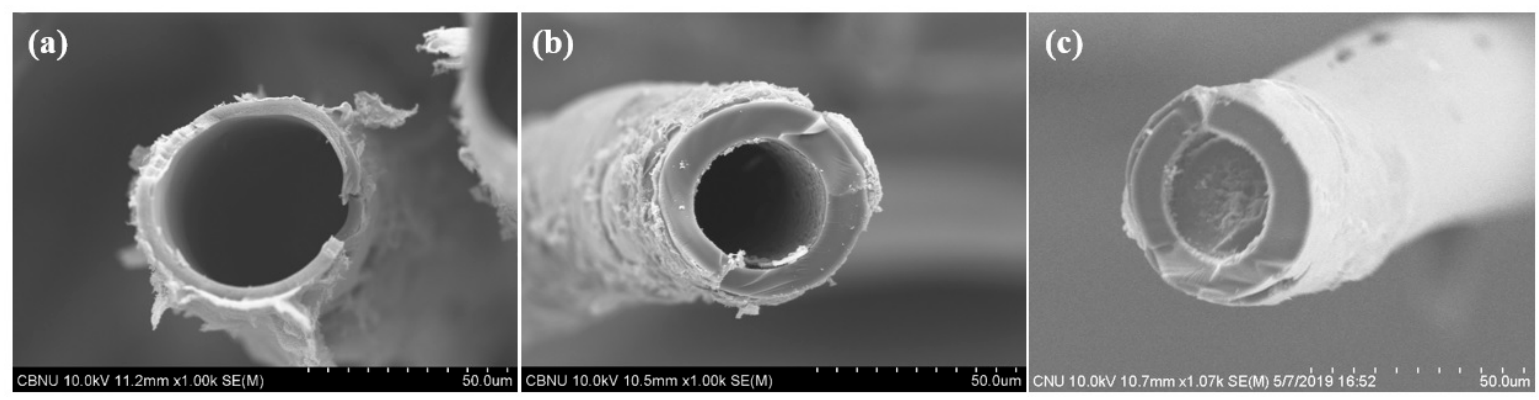

Figure 13. Cross section SEM images after carbonization and activation of the sulfonated LDPE/HDPE sheath-core fibers for time of 60-120 min under $0.25 \mathrm{MPa}$ at $130{ }^{\circ} \mathrm{C}$ : (a) $60 \mathrm{~min}$; (b) $90 \mathrm{~min}$; (c) $120 \mathrm{~min}$.

\section{Conclusions}

In summary, activated LDPE/HDPE sheath-core carbon fibers were manufactured by carbonization and activation with a $\mathrm{KOH}$ solution after cross-linking via sulfonation at various temperatures (120-150 ${ }^{\circ} \mathrm{C}$ ) and durations (60-150 min) under a load of 0.25 MPa. LDPE/HDPE (50/50 wt.\%/wt.\%) sheath-core fibers were fabricated via melt-conjugate spinning at different draw ratios from 1.2 to 1.6. The activated carbon fibers with the sheath-core structure spun at a draw ratio of 1.5 were characterized by considering the orientation, cross-linking, carbonization, and activation of the LDPE/HDPE sheath-core fibers. Consequently, the tensile properties and specific surface area of the activated LDPE/HDPE sheath-core carbon fibers were found to be maximized when cross-linking was carried out at $130{ }^{\circ} \mathrm{C}$ and $0.25 \mathrm{MPa}$ for $120 \mathrm{~min}$. At this condition, a small pore size, high BET surface area, high AI, and high carbonized yield were achieved, as confirmed by SEM, FTIR, DSC, TGA, and BET analyses. In addition, the tensile properties of the activated carbon fibers were improved by the presence of HDPE in the core, which eventually contributed to enhancing the final mechanical properties of the activated LDPE/HDPE sheath-core carbon fibers. Overall, the activated carbon fibers exhibited a high tensile strength of $\sim 455 \mathrm{MPa}$, initial modulus of $\sim 14.4 \mathrm{GPa}$, and BET surface area of $\sim 1224.36 \mathrm{~m}^{2} / \mathrm{g}$. The resulting LDPE/HDPE sheath-core activated carbon fibers could be utilized for various applications such as environmental (air purification filter, water treatment filter), chemical (recovery of organic solvents and compounds), military defense (gas masks and fire-resistant clothing), and next-generation battery materials.

Supplementary Materials: The following are available online at http://www.mdpi.com/2073-4360/12/12/2895/s1, Figure S1: Burning test images of the sulfonated LDPE/HDPE sheath-core fibers for time of 60-120 min under $0.25 \mathrm{MPa}$ at $130{ }^{\circ} \mathrm{C}$.; Table S1: Elementary composition of the LDPE/HDPE sheath-core fibers for various crosslinking times at $130{ }^{\circ} \mathrm{C}$.

Author Contributions: Writing—original draft preparation, J.S.W.; resources and data curation, J.S.W. and H.R.L.; methodology, M.J.L. and M.H.J.; visualization, J.S.W. and H.R.L.; investigation, M.J.L. and M.H.J.; 
resources-funding acquisition-supervision, S.G.L.; project administration, S.G.L. and J.S.W.; conceptualization and writing-review \& editing. Y.L.J. All authors have read and agreed to the published version of the manuscript.

Funding: This research was supported by Source technology development project through the National Research Foundation of Korea (NRF) funded by the Ministry of Education (2018086216).

Conflicts of Interest: The authors declare no conflict of interest.

\section{References}

1. Bagheri, S.; Julkapli, N.M. Effect of hybridization on the value-added activated carbon material. Int. J. Ind. Chem. 2016, 7, 249-264. [CrossRef]

2. Mochida, I.; Korai, Y.; Shirahama, M.; Kawano, S.; Hada, T.; Seo, Y.; Yoshikawa, M.; Yasutake, A. Removal of SOx and NOx over activated carbon fibers. Carbon 2000, 38, 227-239. [CrossRef]

3. Amoros, D.C.; Monge, J.A.; Solano, A.L. Characterization of Activated Carbon Fibers by CO2 Adsorption. Langmuir 1996, 12, 2820-2824. [CrossRef]

4. Mangun, C.L.; Benak, K.R.; Economy, J.; Foster, K.L. Surface chemistry, pore sizes and adsorption properties of activated carbon fibers and precursors treated with ammonia. Carbon 2001, 39, 1809-1820. [CrossRef]

5. Frank, E.; Hermanutz, F.; Buchmeiser, M.R. Carbon fibers: Precursors, manufacturing, and properties. Macromol. Mater. Eng. 2012, 297, 493-501. [CrossRef]

6. Sedghi, A.; Farsani, R.E.; Shokuhfar, A. The effect of commercial polyacrylonitrile fibers characterizations on the produced carbon fibers properties. J. Mater. Process. Technol. 2008, 198, 60-67. [CrossRef]

7. Frank, E.; Steudle, L.M.; Ingildeev, D.; Sporl, J.M.; Buchmeiser, M.R. Carbon fibers: Precursor systems, processing, structure, and properties. Angew. Chem. Int. Ed. 2014, 53, 5262-5298. [CrossRef]

8. Jain, M.K.; Abhiraman, A.S. Conversion of acrylonitrile-based precursor fibres to carbon fibres. J. Mater. Sci. 1987, 22, 278-300. [CrossRef]

9. Wangxi, Z.; Jie, L.; Gang, W. Evolution of structure and properties of PAN precursors during their conversion to carbon fibers. Carbon 2003, 41, 2805-2812. [CrossRef]

10. Yao, Y.; Chen, J.; Liu, L.; Dong, Y.; Liu, A. Mesophase pitch-based carbon fiber spinning through a filter assembly and the microstructure evolution mechanism. J. Mater. Sci. 2014, 49, 191-198. [CrossRef]

11. Chiang, Y.C.; Lee, C.Y.; Lee, H.C. Surface chemistry of polyacrylonitrile- and rayon-based activated carbon fibers after post-heat treatment. Mater. Chem. Phys. 2007, 101, 199-210. [CrossRef]

12. Holmes, M. Lowering the cost of carbon fiber. Reinf. Plast. 2017, 61, 279-283. [CrossRef]

13. Wang, H.; Chen, S.; Zhang, J. Surface treatment of LLDPE and LDPE blends by nitric acid, sulfuric acid, and chromic acid etching. Colloid Polym. Sci. 2009, 287, 541-548. [CrossRef]

14. Kaneko, M.; Kumagai, S.; Nakamura, T.; Sato, H. Study of sulfonation mechanism of low-density polyethylene films with fuming sulfuric acid. J. Appl. Polym. Sci. 2004, 91, 2435-2442. [CrossRef]

15. Younker, J.M.; Saito, T.; Hunt, M.A.; Naskar, A.K.; Beste, A. Pyrolysis pathways of sulfonated polyethylene, an alternative carbon fiber precursor. J. Am. Chem. Soc. 2013, 135, 6130-6141. [CrossRef]

16. Hunt, M.A.; Saito, T.; Brown, R.H.; Kumbhar, A.S.; Naskar, A.K. Patterned functional carbon fibers from polyethylene. Adv. Mater. 2012, 24, 2386-2389. [CrossRef]

17. Jin, Z.; Yan, X.; Yu, Y.; Zhao, G. Sustainable activated carbon fibers from liquefied wood with controllable porosity for high-performance supercapacitors. J. Mater. Chem. A 2014, 2, 11706-11715. [CrossRef]

18. Kim, J.; Lee, J. Preparation of carbon fibers from linear low density polyethylene. Carbon 2015, 94, 524-530. [CrossRef]

19. Meola, C.; Carlomagno, G. Cross-linked polyethylene. Encycl. Chem. Process. 2006, 1, 577-588.

20. Malaika, S.A.; Riasat, S.; Lewucha, C. Reactive antioxidants for peroxide crosslinked polyethylene. Polym. Degrad. Stab. 2017, 145, 11-24. [CrossRef]

21. Ciuprina, F.; Teissedre, G.; Filippini, J. Polyethylene crosslinking and water treeing. Polymer 2001, 42, 7841-7846. [CrossRef]

22. Li, C.; Zhu, H.; Salim, N.; Fox, B.; Hameed, N. Preparation of microporous carbon materials via in-depth sulfonation and stabilization of polyethylene. Polym. Degrad. Stab. 2016, 134, 272-283. [CrossRef]

23. Postema, A.R.; Groot, H.D.; Pennings, A.J. Amorphous carbon fibres from linear low density polyethylene. J. Mater. Sci. 1990, 25, 4216-4222. [CrossRef] 
24. Zhang, D.; Bhat, G.S. Carbon fibers from polyethylene-based precursors. Mater. Manuf. Process. 1994, 9, 221-235. [CrossRef]

25. Zhang, D. Carbon fibers from oriented polyethylene precursors. J. Thermoplast. Compos. Mater. 1993, 6, 38-48. [CrossRef]

26. Penning, J.P.; Lagcher, R.; Pennings, A.J. The effect of diameter on the mechanical properties of amorphous carbon fibres from linear low density polyethylene. Polym. Bull. 1991, 25, 405-412. [CrossRef]

27. Zhang, D.; Sun, Q. Structure and properties development during the conversion of polyethylene precursors to carbon fibers. J. Appl. Polym. Sci. 1996, 62, 367-373. [CrossRef]

28. Li, Y.; Zhang, M. Mechanical properties of activated carbon fibers. In Activated Carbon Fiber and Textiles; Chen, J.Y., Ed.; Woodhead Publishing Series in Textiles; Woodhead Publishing: Cambridge, UK, 2017; pp. 167-179.

29. Karacan, I.; Benli, H. Use of sulfonation procedure for the development of thermally stabilized isotactic polypropylene fibers prior to carbonization. J. Appl. Polym. Sci. 2012, 123, 234-245. [CrossRef]

Publisher's Note: MDPI stays neutral with regard to jurisdictional claims in published maps and institutional affiliations.

(C) 2020 by the authors. Licensee MDPI, Basel, Switzerland. This article is an open access article distributed under the terms and conditions of the Creative Commons Attribution (CC BY) license (http://creativecommons.org/licenses/by/4.0/). 